\title{
In vitro- and in vivo-targeted tumor lysis by an MMP2 cleavable melittin-LAP fusion protein
}

\author{
LORI HOLLE ${ }^{1}$, WEN SONG ${ }^{1}$, ERIC HOLLE $^{1}$, YANGZHANG WEI $^{1,2}$, JINHUA LI ${ }^{1}$, \\ THOMAS E. WAGNER ${ }^{1,2}$ and XIANZHONG YU ${ }^{1,2}$ \\ ${ }^{1}$ Oncology Research Institute of the Greenville Hospital System, Greenville, SC 29605; \\ ${ }^{2}$ Department of Biological Science, Clemson University, Clemson, SC 29634, USA
}

Received May 29, 2009; Accepted July 23, 2009

DOI: 10.3892/ijo_00000396

\begin{abstract}
Chemotherapy is one of the main treatment options for cancer, but the effectiveness of chemotherapeutic drugs is severely limited due to their systemic toxicity. Therefore, the need for a more targeted approach in tumor treatment is obvious. A tumor-activated agent would decrease systemic toxicity as well as increase the efficacy of the treatment. It has previously been shown that the latency of pro-TGF- $\beta$ is conferred by dimerization of two latency-associated peptides (LAP) that form a protective shield, which is cleaved off upon activation by matrix metalloproteinases (MMPs). It has also been shown that the fusion of this LAP peptide with other cytokines can confer their latency. In the present study, a recombinant adenovirus with a fusion gene encoding a tumor-activated pro-cytolytic peptide was made in which the LAP domain of TGF- $\beta$ was fused with melittin, a potent cytolytic toxin, with an MMP2 cleavage site in between the two. In vitro studies show that the melittin-MMP2-LAP recombinant adenovirus can be activated by MMP2 which leads to the release of free melittin to lyse the target cells. In vivo studies show approximately a $70 \%$ decrease in B16 tumor volume in melittin-MMP2-LAP recombinant adenovirustreated mice as compared to control mice. No significant systemic toxicity was observed in the treated mice.
\end{abstract}

\section{Introduction}

Despite the progress in developing new strategies for tumor treatments (1-7), chemotherapy remains the main option for systemic treatment in the majority of cancer patients. The effectiveness of chemotherapeutic agents is, however, severely limited by their systemic toxicity, due to the fact that the majority of clinically used anti-tumor drugs are cytotoxins. These cytotoxins mainly target dividing cancer cells as well

Correspondence to: Dr Xianzhong Yu, Oncology Research Institute, Greenville Hospital System, 900 West Faris Road, Greenville, SC 29605, USA

E-mail: xyu@ghs.org

Key words: fusion protein, lytic peptides, melittin, latencyassociated peptide, matrix metalloproteinase-2, cancer as dividing non-cancerous cells. Therefore, it is obvious that a more tumor-selective agent would decrease the systemic toxicity and improve the overall efficacy. One of the most direct approaches to this objective is to design prodrugs that will be specifically activated and released when they reach the vicinity of a tumor cell or mass (8-10). Transforming growth factor $\beta$ (TGF- $\beta$ ) is unique among cytokines in that it is secreted as a latent cytokine with an N-terminal domaintermed latency-associated peptide (LAP) (11). LAP is cleaved extracellularly and then forms dimers through disulfide binding. The LAP serves as a shell to protect the C-terminal portion of the cytokine from functioning as well as prevent degradation. Upon activation, the active TGF- $\beta$ is released from this shell by proteolytic cleavage of the LAP domain (12). Taking advantage of this natural LAP protective shell, a hybrid IFN- $\beta$ with a LAP domain and an MMP cleavage site has been successfully engineered to target inflammation sites (13). This latent IFN- $\beta$ will only be activated by MMP cleavage. This result indicates that LAP could be used to engineer other latent peptides with varied biological functions (14).

Membrane-disrupting lytic peptides have been studied intensively for experimental tumor treatment due to their potent, mechanical cell membrane-disrupting capability (15). Different types of cytolytic toxins, such as anthrax toxin, aerolysin, two-chain $\alpha$-hemolysin, and hecate have been used in fusion proteins or conjugates to either directly target the tumor cell surface through ligands and receptors, or be activated through tumor-specific proteases, such as urokinase, PSA, cathepsin B and MMPs (16-19). In a previous study, we have shown that an MMP2 cleavable melittin, the poreforming toxin from honey bee, conjugated with avidin can specifically target tumor cells in vitro and in vivo (20). It has also been shown that recombinant adenovirus encoding a melittin gene controlled by an AFP promoter is effective against hepatocellular carcinoma in vivo (21). In the present study, an MMP2 cleavable fusion gene between LAP and melittin was constructed. When delivered through recombinant adenovirus, this latent fusion protein can specifically target tumor cells in vitro and in vivo.

\section{Materials and methods}

Melittin-MMP2-LAP fusion gene synthesis. The melittinMMP2-LAP fusion gene DNA was synthesized by GenScript 
Corp and cloned into plasmid pUC57 at the EcoRV site (melittin-MMP2-LAP/pUC57).

Melittin-MMP2-LAP mammalian expression vector construction. Initially, melittin-MMP2-LAP from the original melittinMMP2-LAP/pUC57 was cloned into multiple cloning site A (MCS A) of pIRES (Invitrogen, Carlsbad, CA). MelittinMMP2-LAP/pUC57 was digested with EcoR1 and SalI and then ligated into pIRES that had been cut with XhoI and EcoR1. This construct was named melittin-MMP2-LAP/ pIRES. Next, dhFr from pSV2-dhfr (ATCC) was cloned into MCS B of melittin-MMP2-LAP/pIRES. dhFr was removed from pSV2-dhFr by digesting with HindIII and NcoI and blunted with Klenow (NEB). The blunted dhFr fragment was then cloned into MCS B of melittin-MMP2-LAP/pIRES which had been digested with Not 1 and SalI, blunted with Klenow, and dephosplorylated with calf intestinal alkaline phosphatase (NEB). This construct was named melittinMMP2-LAP/pIRES/dhFr.

Production of a melittin-MMP2-LAP/CHO cell line. $\mathrm{CHO} / \mathrm{dhFr}$ - cells (CHO duk-) were purchased from ATCC (Manassas, VA) and cultured in DMEM high glucose (Hyclone, Logan, UT) supplemented with $10 \%$ FBS (Hyclone) $4 \mathrm{mM}$ L-glutamine (Gibco, Carlsbad, CA), $0.1 \mathrm{mM}$ hypoxanthine (Gibco), $0.016 \mathrm{mM}$ thymidine (Gibco), and $0.002 \mathrm{mM}$ Methotrexate (Sigma, St. Louis, MO). Cells were grown at $37^{\circ} \mathrm{C}$ in the presence of $5 \% \mathrm{CO}_{2}$. Once the cell line was well established in media containing $10 \%$ FBS the FBS amount was gradually decreased to $1 \%$. Lipofectamine 2000 (Invitrogen) was used for melittin-MMP2-LAP trans-fection into $\mathrm{CHO} / \mathrm{dhFr}$ - cells. Prior to transfections, $\mathrm{CHO} / \mathrm{dhFr}-$ cells were maintained in 1\% FBS DMEM media (no MTX) and were transfected with either Lipofectamine alone or Lipofectamine + melittin-MMP2-LAP $(4 \mu \mathrm{g})$. The transfections were performed according to the manufacturer's protocol. Forty-eight hours post-transfection, the cells were placed under selection by addition of $1 \mathrm{mg} / \mathrm{ml}$ Geneticin (G418; Gibco) to media and removal of HT (hypoxanthine, thymidine) from the media.

Melittin-MMP2-LAP gene amplification in CHO cells with $M T X$. In order to amplify the production of melittin-MMP2LAP, cells were incubated in media containing increasing amounts of MTX (50, 100, 250 and $500 \mathrm{nM})$. At each MTX concentration, cells were incubated for approximately $48 \mathrm{~h}$ before switching to the next higher concentration of MTX.

Protein extraction from cells. Lysate was obtained from both $\mathrm{CHO}$ and melittin-MMP2-LAP/CHO cell lines by the following procedure. T-75 flasks of cells were washed with ice cold PBS followed by incubation with $1 \mathrm{ml}$ cell lysis buffer $(50 \mathrm{mM}$ Tris $\mathrm{pH} 7.4,1 \% \mathrm{NP}-40,0.25 \%$ sodium deoxycholate, $150 \mathrm{mM} \mathrm{NaCl}, 1 \mathrm{mM}$ EDTA, $0.1 \%$ SDS and protease inhibitor cocktail; Complete Mini tablets; Roche, Indianapolis, IN). Cells were incubated approximately 5-10 $\mathrm{min}$ in lysis buffer and lysate was then passed through a 21 gauge needle 5-6X to sheer genomic DNA, incubated on ice $10 \mathrm{~min}$, and centrifuged at $14,000 \mathrm{rpm}$ for $10 \mathrm{~min}$ at $4^{\circ} \mathrm{C}$ in a microcentrifuge. Supernatant was saved for Western analysis.
Analysis of melittin-MMP2-LAP expression in cultured cells. Melittin-MMP2-LAP cell lysate was analyzed for protein expression via Western analysis using anti-human LAP (TGF-ß1) antibody (R\&D). Protein was extracted from cells as described above. Upon extraction, an equal volume of sample $(30 \mu \mathrm{g})$ and $2 \mathrm{X}$ Laemmli loading buffer (Sigma) were mixed, boiled for $5 \mathrm{~min}$, and then analyzed by $15 \%$ SDS-PAGE (Biorad). hLAP (R\&D) was used as a positive control and prestained broad-range protein molecular weight marker (NEB) was used to determine the size of the fusion protein. After gel electrophoresis, protein was transferred to Hybond nitrocellulose membrane, (GE Healthcare; Piscataway, NJ) blocked with 5\% non-fat powdered milk, and incubated with anti-human LAP (TGF- $\beta$ ) antibody (1:400 dilution) overnight at $4^{\circ} \mathrm{C}$. The following day membranes were washed with PBS containing $0.05 \%$ Tween-20 and then incubated with a 1:2000 dilution of a donkey anti-goat HRP-conjugated secondary antibody (Santa Cruz Biotech). Membranes were developed for 1 min using enhanced chemiluminescence reagents (ECL; GE Healthcare).

In vitro cyto-toxicity assay. In order to determine whether or not the melittin-MMP2-LAP fusion protein produced in $\mathrm{CHO}$ cells was active or not, a cell-based assay was performed. On day zero, melittin-MMP2-LAP transfected CHO cells and non-transfected $\mathrm{CHO}$ cells were plated in an opaque walled 96-well tissue culture plate (Fisher) at a cell density of 20,000 cells/well in a volume of $100 \mu 1$. Approximately $24 \mathrm{~h}$ after plating cells, $0.5 \mu \mathrm{g}$ of active MMP2 (Calbiochem; San Diego, CA) was added to the appropriate wells and then cells were incubated for approximately $72 \mathrm{~h}$ at $37^{\circ} \mathrm{C}$ in the presence of $5 \% \mathrm{CO}_{2}$. After $72 \mathrm{~h}$ of MMP2 treatment the CellTiter-Glo kit (Promega, Madison, WI) and the Lumistar (BMG Labtech) were used to determine the cell viability of the samples by measuring the luminescence (RLU) of each sample. The gain was set at 150 to read all the samples. The percent decrease was calculated as follows: (RLU; MMP2 treatment - RLU; no MMP2 treatment)/RLU; no MMP2 treatment) x100.

Recombinant melittin-MMP2-LAP adenovirus production. To produce the recombinant adenovirus, melittin-MMP2LAP was first cloned into the pDC312 shuttle vector (Microbix; Toronto, Canada). Both the shuttle vector and melittin-MMP2-LAP/pIRES (described above) were cut with NheI and EcoR1 and ligated to produce melittin-MMP2LAP/pDC312. Secondly, Ad293 cells obtained from Microbix were co-transfected with melittin-MMP2-LAP/pDC312 and

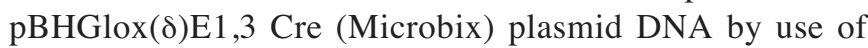
calcium phosphate (Profection mammalian transfection system; Promega). Transfected cells were maintained in normal Ad293 media (DMEM + 10\% FBS) and approximately 1 week after transfection, CPE (cytopathic effect) was visible and then cells were collected and lysed via freeze-thaw cycles. Lysate was tested for the presence of mellitin-MMP2LAP via Western analysis using human LAP antiserum as described above. Recombinant adenovirus was amplified according to standard protocols (BD Adeno-X Expression System1), purified via ViraBind Adenovirus purification kit (Cell Biolabs; San Diego, CA), and tittered by use of QuickTiter Adenovirus Titer Immunoassay Kit (Cell Biolabs). The 
units for the viral titer were IFU/ml. Recombinant virus was then concentrated using 100K MWCO centrifugal filter units (Sartorius Stedim; Edgewood, NY).

Recombinant melittin-LAP (MMP2 del.) adenovirus production. In order to make an MMP2 cleavage control adenovirus, another recombinant adenovirus was produced in which the MMP2 cleavage site was deleted; melittin-LAP (MMP2 del.). The MMP2 cleavage site was deleted by using inverse PCR. Melittin-MMP2-LAP/pDC312 was used as the template and the following primers synthesized by Operon Biotechnologies Inc. (Huntsville, AL) were used in the PCR reaction; leftmuta (GCCGCCCTGCTGTCTCTTGC) and rightmuta (GGCGGCCTGAGCACCTGTAAGA). Both primers were phosphorylated at their 5 prime ends and SDSPAGE purified. The protocol from the Phusion Site-Directed Mutagenesis Kit obtained from NEB was followed for the Inverse PCR reaction. After Inverse PCR the product was gel-purified using the QIAquick gel purification kit from Qiagen. The PCR product was then circularized with Quick T4 DNA ligase (NEB) in a 5 min reaction and following ligation was transformed into TOP 10 cells (Invitrogen). Several of the transformants were analyzed and it was confirmed by sequencing that the MMP2 cleavage site had been deleted. This recombinant adenovirus was then amplified, purified and tittered as described in the above section titled 'melittin-MMP2-LAP recombinant adenovirus production'.

In vitro cytotoxicity assay using melittin-MMP2-LAP recombinant adenovirus. In order to perform in vitro cytotoxicity assays with the recombinant adenovirus, DU145 cells were plated at a cell density of 5,000 cells/well in an opaque-walled 96-well plate. The volume of cells in each well was $100 \mu \mathrm{l}$. After $24 \mathrm{~h}, \mathrm{DU}-145$ cells were infected with either AD5.CMV LacZ control adenovirus (Quantum Biotechnologies, Montreal, Canada), melittin-LAP (MMP2 del.) recombinant adenovirus, or melittin-MMP2-LAP recombinant adenovirus at a multiplicity of infection (MOI; infectious units/cell) of $0,100,500$ and 1000. Cells were treated with virus for approximately $72 \mathrm{~h}$ and the CellTiter-Glo luminescent cell viability assay (Promega) was then done according to the vendor's protocol. Luminescence values for each sample were measured by use of the Lumistar (BMG Labtech) and the gain was set at 150 . Decrease was calculated using the following equation: (RLU; melittin recombinant adenovirus or RLU; deleted cleavage site - RLU; Ad5.CMV LacZ adenovirus)/RLU; Ad5.CMV LacZ adenovirus) x100.

In vivo tumor killing. In order to determine the effectiveness of the melittin-MMP2-LAP adenovirus in vivo, 10 C57bl6 female mice (Jackson Lab, Bar Harbor, ME) approximately 8 weeks of age were inoculated with $0.5 \times 10^{6} \mathrm{~B} 16$ melanoma cells s.c. Prior to mouse inoculation, B16 tumor cells were obtained from ATCC and cultured in DMEM supplemented with $10 \%$ fetal bovine serum and $50 \mu \mathrm{g} / \mathrm{ml}$ gentamicin (Gibco). The mice used for this study were housed according to the rules of the Institutional Animal Care and Use Committee. Eight days following B16 tumor inoculation 5/10 mouse tumors were injected with $100 \mu 1\left(2.5 \times 10^{8}\right.$ IFU) melittin-MMP2-LAP adenovirus and 5/10 mice were injected

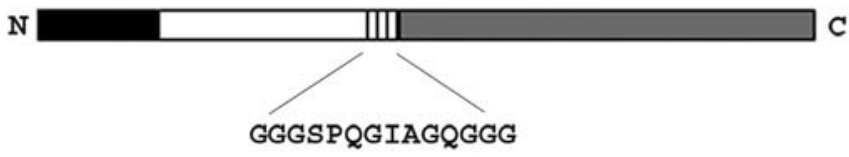

Figure 1. Schematic representation of the primary linear structure of the fusion protein. The black box at the $\mathrm{N}$ terminal represents amino acids 1-29 of the TGF- $\beta$ secretion signal peptide, the white box depicts the complete melittin sequence, and the grey box represents AA 30-273 of the LAP sequence. The box with the vertical lines shows the flexible MMP2 cleavage site.

with an equal amount of AD5. CMV LacZ control virus (Quantum Biotechnologies). Mice were injected twice a week for a total of 5 injections. Prior to each injection mice were weighed and tumor volume was measured $\left(w^{2} x L / 2\right)$. At the end of the study, mice were sacrificed, tumors were removed and weighed.

Statistical analysis. All statistical analyses were performed using GraphPad Prism software. The unpaired t-test was used to calculate P-values. Data were considered significant at $\mathrm{P}<0.05$.

\section{Results}

Construction of melittin-MMP2-LAP fusion gene. The melittin-MMP2-LAP fusion gene was synthesized by GenScript Corp and a schematic of the melittin-MMP2-LAP fusion gene is shown in Fig. 1. The fusion gene is approximately 942 base pairs in size and consists of 4 main segments. The first segment encodes amino acids 1-29 of the original TGF- $\beta$ secretion signal peptide, followed by the complete melittin sequence, and a flexible MMP2 cleavage site (GGGSPQGIAGQGGG). The final segment of the fusion gene encodes amino acids 30-273 of the latency-associated peptide (LAP) domain of TGF- 3 .

Expression of melittin-MMP2-LAP fusion protein in CHO/ dhFr-cells. CHO/dhFr- cells were chosen for melittinMMP2-LAP fusion protein production. $\mathrm{CHO} / \mathrm{dhFr}-$ cells were transfected with melittin-MMP2-LAP/pIRES/dhFr plasmid DNA and were selected by G418 addition (final concentration $1 \mathrm{mg} / \mathrm{ml}$ ) as well as HT depletion. Expression of the melittin-MMP2-LAP fusion protein is shown in Fig. 2. The predicted molecular weight of the melittin-MMP2-LAP fusion protein is approximately $35 \mathrm{kDa}$. A protein approximately this size is shown by Western analysis with hLAP antibody in the melittin-MMP2-LAP transfected cells, but not in the untransfected $\mathrm{CHO}$ cells.

Amplification of melittin-MMP2-LAP fusion gene via MTX. In the $\mathrm{CHO} / \mathrm{dhFr}$ - cell system for protein expression, MTX can be used for gene amplification and therefore increased melittin-MMP2-LAP protein production in CHO cells. Fig. 3 shows that the production of the melittin-MMP2-LAP fusion protein was amplified by use of MTX. Normally the melittinMMP2-LAP CHO cell line is cultured in the presence of 100 nM MTX. By gradually increasing the concentration of 


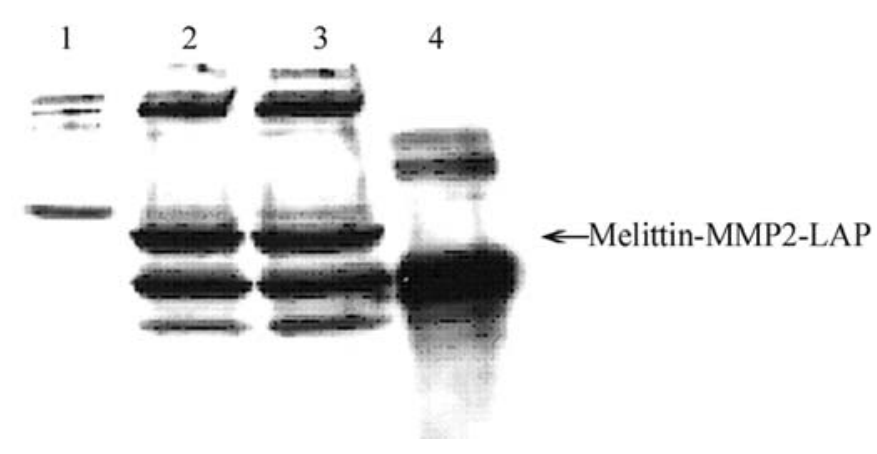

Figure 2. Fusion protein-transfected $\mathrm{CHO}$ cells show expression of a protein with the predicted molecular weight of melittin-MMP2-LAP. Protein was extracted from melittin-MMP2-LAP-transfected and non-transfected CHO cells. Total protein $(30 \mu \mathrm{g})$ was analyzed via SDS-PAGE followed by Western blotting with hLAP antiserum. This antibody will detect the LAP portion of the fusion protein. Protein of the predicted molecular weight $(35 \mathrm{kDa})$ of the fusion protein is detected with hLAP antiserum. This protein is seen only in transfected cells (lanes 2 and 3), not in untransfected $\mathrm{CHO}$ cells (lane 1). Protein analyzed in lanes 2 and 3 was obtained from different tissue culture flasks of cells. hLAP (lane 4) was used as a positive control for Western analysis and has a predicted molecular weight of $27 \mathrm{kDa}$.

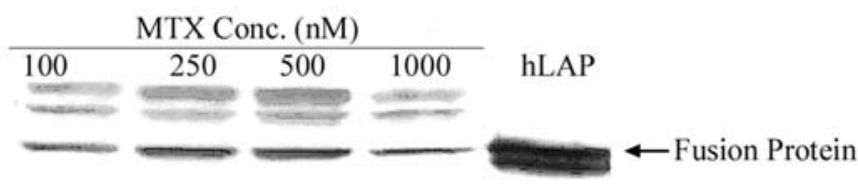

Figure 3. Melittin-MMP2-LAP fusion protein expression is induced in transfected $\mathrm{CHO}$ cells upon addition of methotrexate. The melittin fusion protein-transfected cells were cultured in media containing different amounts of MTX. Cells were exposed to a particular concentration of MTX for $48 \mathrm{~h}$ and then protein was extracted and analyzed via Western analysis with hLAP antiserum. It can be seen in the figure that as the concentration of MTX is increased the amount of the fusion protein also increases. The protein increase is greatest at $500 \mathrm{nM}$ MTX and then starts to decrease at $1000 \mathrm{nM}$ MTX. hLAP was used as a positive control for Western analysis.

MTX in the cells from 100 to $1000 \mathrm{nM}$ MTX, melittinMMP2-LAP fusion protein expression could be increased as shown by the Western analysis in Fig. 3. Protein expression is increased in samples incubated with up to $500 \mathrm{nM}$ MTX. At a concentration higher than $500 \mathrm{nM}$ MTX, for example $1000 \mathrm{nM}$, protein production is decreased.

Cell death is increased in melittin-MMP2-LAP-transfected CHO cells treated with MMP2 as compared to untransfected CHO cells. Fig. 4 represents data obtained from an in vitro cell viability assay. This assay was performed to determine whether or not the protein secreted from the transfected cells was functional. The protein is considered functional if in its secreted form it can be cleaved by MMP2. Once the fusion protein is cleaved, the melittin portion of the peptide will interact with the MMP2 secreting cancer cells and kill them. To activate the fusion protein, both $\mathrm{CHO}$ and melittin-MMP2LAP-transfected CHO cells (50nM MTX) were treated with active MMP2 for $72 \mathrm{~h}$. After MMP2 treatment, cell viability assays were done to determine the percent decreases in luminescent signal (RLU values) in MMP2-treated-transfected and non-transfected cells as compared to cells with no MMP2

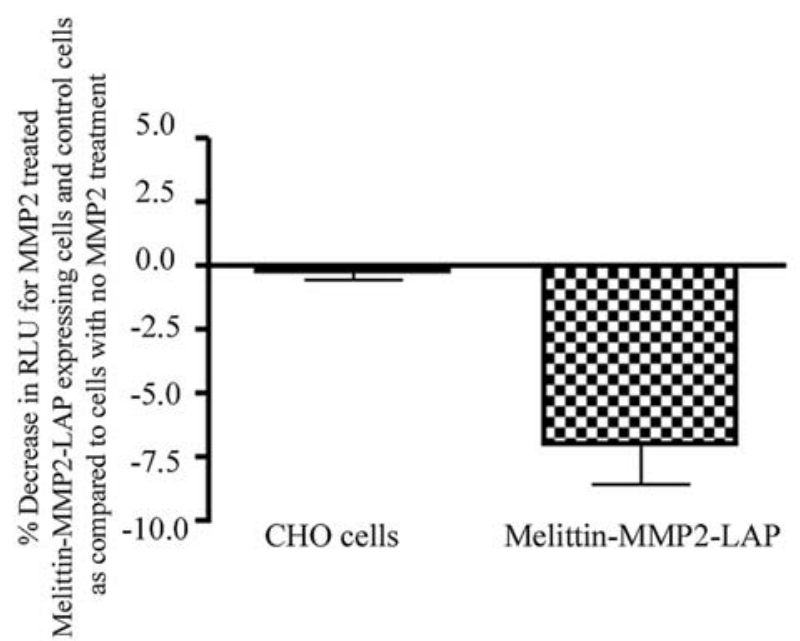

Figure 4. MMP2-treated fusion protein-transfected cells show decreased cell viability as compared to control $\mathrm{CHO}$ cells. Control $\mathrm{CHO}$ cells and melittinMMP2-LAP-transfected CHO cells were plated in a 96-well plate at a cell density of 20,000 cells/well. Control CHO cells were plated in normal $\mathrm{CHO}$ media and the transfected $\mathrm{CHO}$ cells were plated in $50 \mathrm{nM}$ MTX media. Cells were incubated approximately $24 \mathrm{~h}$ at $37^{\circ} \mathrm{C}$ in the presence of $5 \% \mathrm{CO}_{2}$. After $24 \mathrm{~h}, 0.5 \mu \mathrm{g}$ of active MMP2 was added to the cells and the cells were then incubated for $72 \mathrm{~h}$ at $37^{\circ} \mathrm{C}$ and $5 \% \mathrm{CO}_{2}$. The CellTiter-Glo assay was done to determine the luminescent signal (RLU) of each sample. The figure shows the percent decrease in RLU for both the MMP2-treated control CHO cells and the melittin-MMP2-LAP-transfected CHO cells. The percent decrease was calculated as described in the 'Materials and methods' section. The melittin-MMP2-LAP-transfected CHO cells show a much greater percent decrease in cell viability as compared to the control $\mathrm{CHO}$ cells $(n=4$, $\mathrm{p}=0.56)$.

treatment. The luminescent signal (RLU) correlates with the viability of the cells and therefore, as RLU values increase, cell viability also increases. It can be seen from Fig. 4 that the melittin-MMP2-LAP-transfected $\mathrm{CHO}$ cells show a greater $\%$ decrease in RLU as compared to untransfected $\mathrm{CHO}$ cells. These data demonstrate that most likely the fusion protein secreted from the transfected cells is functional since it is cleaved by MMP2. Cleaved peptide will attack the cells, decrease cell viability, and therefore result in a lower luminescent signal as compared to untransfected $\mathrm{CHO}$ cells.

Production of recombinant Melittin-MMP2-LAP adenovirus. To produce the recombinant adenovirus, Ad293 cells were co-transfected with melittin-MMP2-LAP/pDC312 shuttle vector plasmid DNA and pBHGlox( $\delta)$ E1,3 Cre plasmid DNA for recombinant adenovirus production as described by the manufacturer's protocol (Microbix). Recombinant protein production was demonstrated by Western analysis using hLAP antiserum as shown in Fig. 5. Protein with the predicted molecular weight of melittin-MMP2-LAP is only seen in Ad293 cells transfected with melittin-MMP2-LAP (lane 3), not in untransfected cells (lane 1) or Ad5.CMV LacZ infected Ad293 cells (lane 2).

Infection of MMP2-secreting DU-145 cells with the melittin$M M P 2-L A P$ recombinant adenovirus leads to cell lysis. The MMP2-secreting DU-145 prostate cancer cell line was used to determine the effect of the recombinant melittin-MMP2LAP adenovirus on cell viability. Cells were infected with 


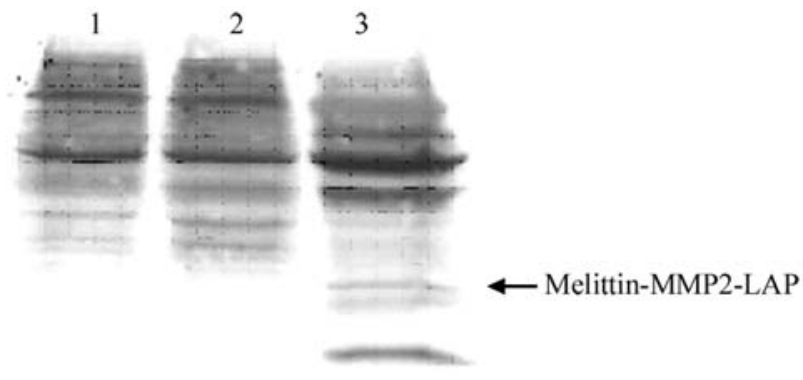

Figure 5. Melittin-MMP2-LAP recombinant adenovirus was successfully produced in Ad293 cells. Ad293 cells were infected with the melittinMMP2-LAP construct as described in the 'Materials and methods' section or Ad5.CMV LacZ. In the above figure equal amounts of protein $(30 \mu \mathrm{g})$ were analyzed by $15 \%$ SDS-PAGE followed by Western blotting with hLAP antibody. It can be seen that a protein of the predicted MW of the melittin-MMP2-LAP fusion protein is seen in the melittin-MMP2-LAPinfected cells (lane 3). This protein is not seen in the Ad293 cells (lane 1) or the Ad293 cells infected with Ad5.CMV LacZ (lane 2).

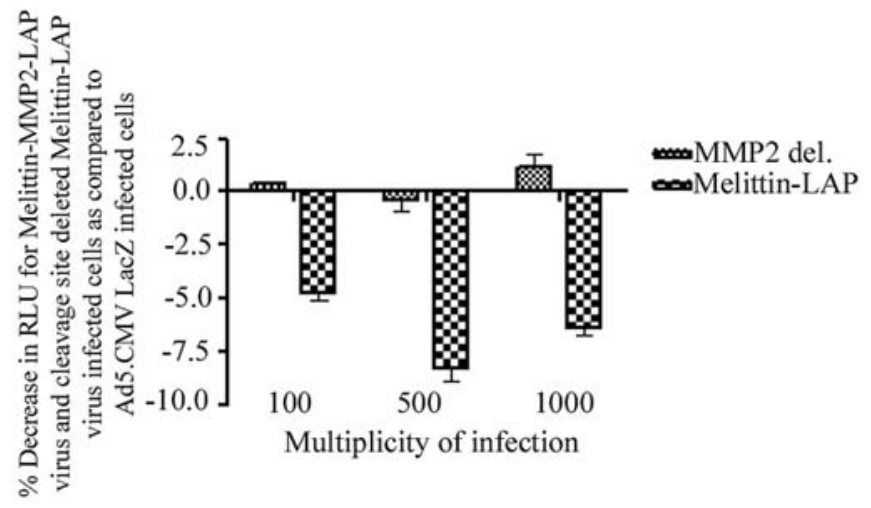

Figure 6. A decrease in cell viability was seen in DU-145 prostate cancer cells infected with the melittin-MMP2-LAP recombinant adenovirus. DU145 cells were plated at a cell density of 5,000 cells/well in a volume of $100 \mu 1$ in a 96-well plate. Cells were incubated overnight at $37^{\circ} \mathrm{C}$ in the presence of $5 \% \mathrm{CO}_{2}$. Cells were then infected for $72 \mathrm{~h}$ with different MOIs of either melittin-MMP2-LAP recombinant adenovirus, melittin-LAP (MMP2 del.) recombinant adenovirus, or AD5.CMV LacZ control adenovirus. Cell viability was then analyzed in these cells by use of the CellTiter-Glo luminescent cell viability assay. The luminescent signal (RLU) was measured by use of the Lumistar and the gain was set at 150 . The data are presented as a \% decrease in RLU for DU-145 cells infected with either melittin-MMP2-LAP recombinant adenovirus or melittin-LAP (MMP2 del.) as compared to cells infected with the same MOI of AD5.CMV LacZ control adenovirus. Overall, cells treated with the melittin recombinant adenovirus show a significant \% decrease in RLU and therefore a decrease in cell viability as compared to the MMP2 cleavage site-deleted adenovirus. Pvalues for data obtained at a MOI of 100, 500 and 1000 are $0.004,0.01$ and 0.007 , respectively.

different MOI (multiplicity of infection) of either melittinMMP2-LAP, melittin-LAP (MMP2 del.), or the Ad5.CMV LacZ recombinant adenovirus. The MMP2 deletion adenovirus was used as a control for the functionality of the MMP2 cleavage site and the Ad5.CMV LacZ recombinant adenovirus was used as the control virus. After $72 \mathrm{~h}$ of infection, Fig. 6 shows that the level of cell viability is decreased in the melittin-MMP2-LAP virus-infected cells thus resulting in a greater \% decrease in RLU values as compared to the melittin-LAP- (MMP2 del.) infected cells. The RLU values
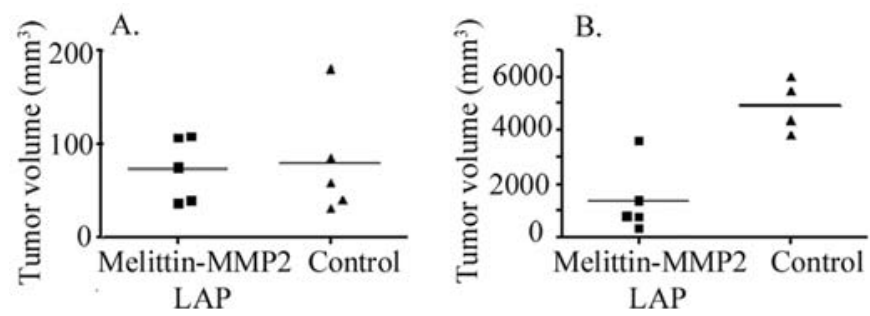

Figure 7. Melittin-MMP2-LAP recombinant adenovirus intratumor injection significantly decreases B16 tumor volume. Ten C57 mice were inoculated with 0.5 million B16 cells s.c. Eight days following tumor inoculation, $100 \mu 1$ of $2.5 \times 10^{8}$ IFU of AD5.CMV LacZ or melittin-MMP2-LAP recombinant adenovirus was injected s.c. into the tumor inoculation site. Mice were injected a total of 5 times. Injections were given $2 x /$ week. (A) The tumor volume of the mice 10 days following tumor inoculation in both groups was approximately the same. (B) Approximately 3 weeks following tumor inoculation and after 4 adenovirus injections the tumor volume in the melittinMMP2-LAP recombinant adenovirus injected mice was approximately $70 \%$ lower as compared to the control adenovirus injected mice. Also one of the mice in the control group had died.

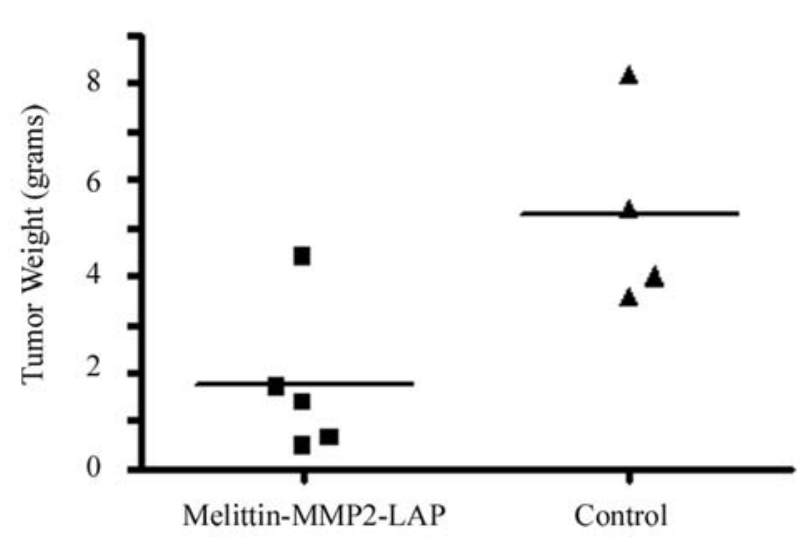

Figure 8. Tumor weight in melittin-MMP2-LAP recombinant adenovirustreated mice is significantly lower as compared to control mice. After 4 recombinant adenovirus injections and approximately 3 weeks after tumor inoculation, mice were sacrificed and tumors were removed. The weight of each tumor was determined. As seen in the figure tumors obtained from mice treated with the melittin-MMP2-LAP recombinant adenovirus are significantly smaller than tumors obtained from control mice.

obtained at a specific MOI for both the melittin-MMP2-LAP and melittin-LAP (MMP2 del.) adenoviruses are directly compared to the RLU value for Ad5.CMV LacZ-infected cells. At a MOI of 100, 500 and 1000, there are significant decreases $(\mathrm{P}<0.5)$ in cell viability in the melittin-MMP2LAP-infected cells as compared to the cleavage site deleted form of the adenovirus. The calculation for $\%$ decrease is described in the 'Materials and methods' section.

Melittin-MMP2-LAP recombinant adenovirus reduces tumor size in vivo. In order to determine the effects of the melittinMMP2-LAP adenovirus in vivo, intratumor injections using the recombinant adenovirus were done in mice bearing B16 tumors. Ad5.CMV LacZ was used for the control intratumor injections. Fig. 7A shows that at the start of intratumor injections, the average tumor volume in both groups was about the same. After 5 injections (2.5 week period), there is a significant decrease ( $70 \%$ decrease) in tumor volume in 
melittin-MMP2-LAP virus-treated mice as compared to Ad5. CMV LacZ-treated mice (Fig. 7B; $\mathrm{P}=0.003$, $\mathrm{n}=5$ ). At the end of the study, mice were sacrificed, tumors were removed from mice, and weighed. The melittin-MMP2-LAP-treated mice showed approximately a $70 \%$ reduction in tumor mass as compared to control mice as demonstrated in Fig. $8(\mathrm{P}=0.02$, $\mathrm{n}=5)$.

\section{Discussion}

Various strategies have been studied to design tumor-targeted lytic peptides (15). These peptides are tumor selective, fast acting, and less likely to develop resistance. In the present study, the very potent mammalian cell lytic melittin was converted into a latent peptide by fusing it with the LAP domain of TGF- $\beta$. The latent melittin can be reactivated with active MMP2 since an MMP2 cleavage site was engineered between the melittin sequence and the LAP domain. The data presented in this paper demonstrate the following: $\mathrm{CHO}$ cells transfected with melittin-MMP2-LAP expression vector were lysed when active MMP2 was added into the culture; recombinant adenovirus encoding the melittin-MMP2-LAP fusion gene lysed DU-145 human prostate tumor cells in vitro, which has been shown to secret active MMP2 (20); and direct intratumoral injection of this recombinant adenovirus significantly reduced the tumor burden.

Previous studies have shown that the fusion of IFN with the LAP domain on its N-terminal can form a better shell as it is found in the native TGF- $\beta$ (15). In the present study, fusion of melittin at its C-terminal resulted in a latent lytic peptide, which will regain its cytolytic activity once cleaved by MMP2. When melittin was fused at its N-terminal with LAP, the fusion protein had no lytic activity, but melittin also lost its lytic activity after it was released from the fusion protein by MMP2 (data not shown). This is probably caused by the extra amino acid residues left from the MMP2 cleavage site since the hydrophobic N-terminal residues are critical for the formation of the helical structure as well as the activity of melittin (17).

Lytic peptides are known for their fast-acting and shortsystemic half-life (15). It has been shown in previous work with cytokines that IFN fusion with LAP has a longer halflife (13). By fusion with the LAP domain, melittin could have a longer half-life, especially if delivered via recombinant adenovirus, but still behave as a fast-acting compound once released by MMP2 cleavage. This could be beneficial in terms of keeping a constant pressure on the tumor but avoiding the risk of building up drug resistance like regular chemotherapeutics.

TGF- $\beta$ has been shown to be overexpressed in human tumors (22-25). The recombinant proregion of TGF- $\beta$ exhibited antagonist activity against active TGF- $\beta$ in a transgenic mouse model (26). The LAP domain cleaved from the fusion protein could be another factor generated in the present study to have anti-tumor activity. This could be answered in the future with a LAP domain only virus as the control.

MMP-2 and other MMPs play a very important role in normal tissue-remodeling events such as embryonic development, angiogenesis, ovulation, mammary gland involution and wound healing. MMP2 is also involved in osteoblastic bone formation and/or inhibits osteoclastic bone resorption (27). This will certainly limit the use of the current approach under those physiological conditions. Although the in vivo data from the current study suggest that the experimental mice tolerated well the treatments, any prediction on the possible side-effects of the present study has to be cautious mainly because the recombinant adenovirus was injected directly into the tumor mass and the lytic peptide has a very fast kinetics of action. These two factors can certainly prevent meaningful amounts of the fusion peptide produced inside the tumor from leaking out and activating somewhere else.

\section{References}

1. Mach N and Dranoff G: Cytokine-secreting tumor cell vaccines. Curr Opin Immunol 12: 571-575, 2000.

2. Rossowska J and Pajtasz-Piasecka E: Dendritic cells and their applications in cancer immunotherapy - achievements and future prospects. Postepy Hig Med Dosw 57: 501-518, 2003.

3. Desai SB and Libutti SK: Tumor angiogenesis and endothelial cell modulatory factors. J Immunother 22: 186-211, 1997.

4. Brower V: Tumor angiogenesis - new drugs on the block. Nat Biotechnol 17: 963-968, 1999.

5. Perry PJ, Gowan SM, Read MA, Kelland LR and Neidle S: Design, synthesis and evaluation of human telomerase inhibitors based upon a tetracyclic structural motif. Anticancer Drug Des 14: 373-382, 1999.

6. Fridman WH and Tartour E: The use of cytokines in the treatment of solid tumours. Hematol Cell Ther 39: 105-108, 1997.

7. Trail PA and Bianchi A: Monoclonal antibody drug conjugates in the treatment of cancer. Curr Opin Immunol 11: 584-588, 1999.

8. Denny WA: Tumor-activated prodrugs - a new approach to cancer therapy. Cancer Invest 22: 604-619, 2004.

9. Chari RVJ: Targeted delivery of chemotherapeutics: tumoractivated prodrug therapy. Adv Drug Deliv Rev 31: 89-104, 1998.

10. Sinhababu AK and Thakker DR: Prodrugs of anticancer agents. Adv Drug Deliv Rev 19: 241-273, 1996.

11. Kingsley DM: The TGF- $\beta$ superfamily: new members, new receptors, and new genetic tests of function in different organisms. Genes Dev 8: 133-146, 1994.

12. Taipale J, Koli K and Keski-Oja J: Release of transforming growth factor-beta 1 from the pericellular matrix of cultured fibroblasts and fibrosarcoma cells by plasmin and thrombin. $\mathbf{J}$ Biol Chem 267: 25378-25384, 1992.

13. Adams G, Vessillier S, Dreja H and Chernajovsky Y: Targeting cytokines to inflammation sites. Nat Biotechnol 21: 1314-1320, 2003.

14. Nissim A, Gofur Y, Vessillier S, Adams G and Chernajovsky Y: Methods for targeting biologicals to specific disease sites. Trends Mol Med 10: 269-274, 2004.

15. Leuschner $\mathrm{C}$ and Hansel W: Membrane disrupting lytic peptides for cancer treatments. Curr Pharm Des 10: 22992310, 2004.

16. Abi-Habib RJ, Singh R, Liu S, Bugge TH, Leppla SH and Frankel AE: A urokinase-activated recombinant anthrax toxin is selectively cytotoxic to many human tumor cell types. Mol Cancer Ther 5: 2556-2562, 2006.

17. Williams SA, Merchant RF, Garrett-Mayer E, Isaacs JT, Buckley JT and Denmeade SR: A prostate-specific antigenactivated channel-forming toxin as therapy for prostatic disease. J Natl Cancer Inst 99: 376-385, 2007.

18. Panchal RG, Cusack E, Cheley S and Bayley H: Tumor protease-activated, pore-forming toxins from a combinatorial library. Nat Biotechnol 14: 852-856, 1996.

19. Hansel W, Leuschner C and Enright F: Conjugates of lytic peptides and LHRH of BCG target and cause necrosis of prostate cancers and metastases. Mol Cell Endocrinol 269: 2633, 2007.

20. Holle L, Song W., Holle E, Wei Y, Wagner T and Yu X: A matrix metalloproteinase 2 cleavable melittin/avidin conjugate specifically targets tumor cells in vitro and in vivo. Int J Oncol 22: 93-98, 2003 . 
21. Ling CZ, Li B, Zhang C, Zhu DZ, Huang XQ, Gu W and Li SX: Inhibitory effect of recombinant adenovirus carrying melittin gene on hepatocellular carcinoma. Ann Oncol 16: 109-115, 2005.

22. Werkmeister JA, Kirkpatrick A, McKenzie JA and Rivett DE: The effect of sequence variations and structure on the cytolytic activity of melittin peptides. Biochim Biophys Acta 1157: 50-54, 1993.

23. Barrett-Lee P, Travers M, Luqmani Y and Coombes RC: Transcripts for transforming growth factors in human breast cancer: clinical correlates. Br J Cancer 61: 612-617, 1990.

24. Coffey RJ Jr, Shipley GD and Moses HL: Production of transforming growth factors by human colon cancer lines. Cancer Res 46: 1164-1169, 1986.
25. Eastham JA, Truong LD, Rogers E, Kattan M, Flanders KC, Scardino PT, et al: Transforming growth factor-beta 1: comparative immunohistochemical localization in human primary and metastatic prostate cancer. Lab Invest 73: 628-635, 1995.

26. Bottinger EP, Factor VM, Tsnag ML, Weatherbee JA, Kopp JB, Qian SW, et al: The recombinant proregion of transforming growth factor beta 1 (latency-associated peptide) inhibits active transforming growth factor beta 1 in transgenic mice. Proc Natl Acad Sci USA 93: 5877-5882, 1996.

27. Nagase H and Woessner JF: Matrix Metalloproteinases. J Biol Chem 274: 21491-21494, 1999. 